\title{
An Efficient Space-Time Coding Scheme for Time Dispersive MIMO Channels
}

\author{
Salim Abdelkareem Alkhawaldeh \\ Electrical Engineering Department, Faculty of Engineering Technology, Albalqa Applied University, Amman, Jordan \\ Email: skhawaldeh@yahoo.com
}

Received January 24, 2012; revised February 14, 2012; accepted March 12, 2012

\begin{abstract}
In this paper, we introduce an efficient space-time coding scheme for time dispersive Multiple-Input Multiple-Output (MIMO) channels. Channel layering and Orthogonal Frequency Division Multiplexing (OFDM) technique are used in the proposed scheme. The proposed scheme is based on maximizing the distance between any two codewords. This is done by inserting an optimized phase shifts between the symbols in the same layer and between different layers. This way leads to the increase of the achieved diversity and coding gains. As a result, the performance of the system will be improved. Simulation results show the efficiency of the proposed scheme compared to the conventional schemes.
\end{abstract}

Keywords: OFDM; Fading; Space-Time Coding; Diversity; MIMO

\section{Introduction}

MIMO systems have taken a great deal of research interest. This technology leads to a significant improvement in the performance and bandwidth efficiency of the wireless system. The increase of the performance is due to that MIMO system provides space diversity which plays important role to combat the time-varying multipath fading caused by wireless channels. The improvement in the bandwidth efficiency is due to that the channel capacity of the MIMO system is equal to that of the Single-Input Single-Output (SISO) system times the number of transmit or receive antennas, which is smallest $[1,2]$.

A number of space-time trellis codes have been proposed for narrow and wide band channels [3,4]. These codes suffer from decoding complexity especially when the number of antennas increases. To decrease the decoding complexity, space-time block codes have been presented for narrow and wide band channels [5-8]. A disadvantage of these codes is the degradation of the performance compared to space-time trellis codes. In recent years, there is a big demand on the multi-rate wireless services. Therefore, a number of multi-rate spacetime coding schemes have been proposed [9-13]. A prominent drawback of these codes is that distance between some codewords is very small which yields to a significant degradation in the performance.

To overcome this problem, in this paper, we propose a two-layer space-time coding scheme for time dispersive MIMO system. The idea behind the proposed scheme is that the minimum distance between any two codewords is maximized which leads to the improvement in the diversity and coding gains. As a result, the performance of our scheme increases compared to the conventional schemes. Simulation results are provided to show the significant improvement in the performance achieved by this scheme.

In Section 2, we describe the system model of OFDM space-time coding system with two transmit and two receive antennas. The proposed coding scheme is presented in Section 3. We introduce and discuss simulation results in Section 4 to show the validity and efficiency of the proposed scheme. Finally, conclusions are presented in Section 5.

\section{System Model}

Consider a wireless communication system equipped with two transmit and two receive antennas under the assumption of quasi-static fading channels. At a given time $k$, signal $u_{i}(k)$ is transmitted from antenna $i$, whereas signal $y_{j}(k)$ is received at antenna $j$. Let $h_{j i}(l)$ be the gain of the $l^{\text {th }}$ resolvable path from transmit antenna $i$ to receive antenna $j$. Under the assumption of quasi-static fading, $h_{j i}(l)$ will be constant over the duration of a frame but different from frame to frame. Furthermore, the path gains are assumed to be samples of zero-mean complex Gaussian random variables that are mutually independent, i.e., $E\left[h_{j i}^{*}(k) h_{m, n}(s)\right]=0$ when $(j, i, k) \neq(m, n, s)$.

If there are $L$ resolvable paths for each channel, the received signal $y$ due to $K$ transmitted signals from each 
transmit antenna can be written as

$$
\mathbf{y}=\left[\begin{array}{c}
y_{1}(0) \\
y_{1}(K+L-2) \\
y_{2}(0) \\
\vdots \\
y_{2}(K+L-2)
\end{array}\right]=\mathbf{H}\left[\begin{array}{c}
u_{1}(0) \\
\vdots \\
u_{1}(K-1) \\
u_{2}(0) \\
\vdots \\
u_{2}(K-1)
\end{array}\right]+\mathbf{n}
$$

where $\boldsymbol{n}$ is the complex white Gaussian noise with zero mean and variance $\boldsymbol{N}_{o} \boldsymbol{I}$ and the channel matrix $\mathbf{H}$ with dimensions $2(K+L-1) \times 2 K$ is given by

$$
\mathbf{H}=\left[\begin{array}{ll}
\mathbf{H}_{11} & \mathbf{H}_{12} \\
\mathbf{H}_{21} & \mathbf{H}_{22}
\end{array}\right]
$$

with

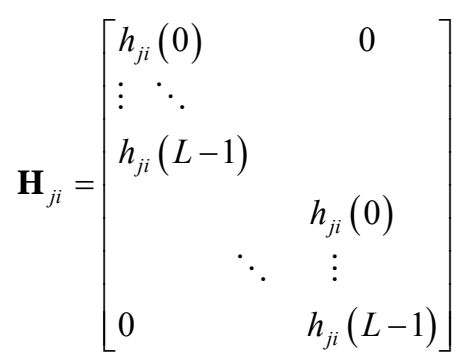

Since the space-time codes introduced in [9] were designed for flat fading channels, they cannot be directly employed for frequency-selective channels $(L>1)$. One way to extend the space-time codes to frequency-selective channels is through the use of OFDM. OFDM technique mathematically changes the slow frequency selective fading channel to multi flat fading channels. Figure 1 shows a baseband representation of the conventional
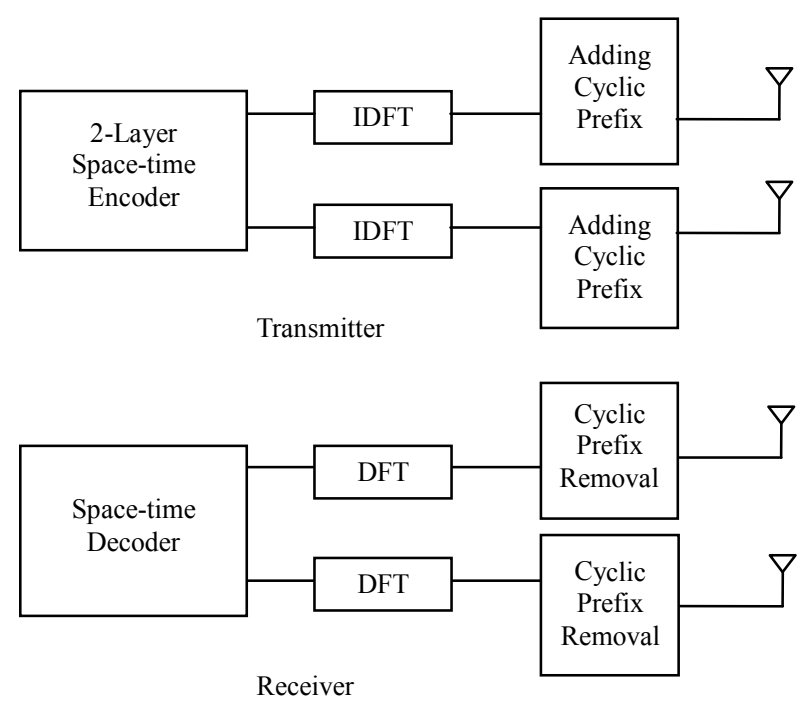

Figure 1. OFDM space-time coding system with two transmit and two receive antennas. two-layer Linear Dispersion Codes (LDC) [9] combined with OFDM system. In this system, by adding a cyclic prefix (CP) to each frame of the signal at the transmitter and removing it at the receiver, the channel matrix becomes block circulant. Then OFDM technique is employed to transform the frequency-selective fading channel into a set of parallel flat sub-channels so that the space-time codes in [9] can be used.

However, Hassibi scheme [9] does not fully exploit the frequency diversity introduced by the existence of multiple resolvable paths. Also, it does not achieve maximized coding gain. Although, the space time coding scheme proposed by Xiaoli Ma [10] achieves full diversity order but the minimum distance between any two codewords is not maximized which leads to significant degradation in the coding gain. Therefore, in the next section we present a new space-time coding scheme combined with OFDM for wide band wireless systems that maximizes both the diversity order and coding gain.

\section{Proposed Space-Time Coding Scheme for Time Dispersive MIMO Channels}

In this section, we propose an efficient space-time coding scheme with two layers for time dispersive MIMO channels. The encoding of our scheme is applied block by block. Each block has $T N$ symbol. The input block to the two-layer space-time encoder is $\boldsymbol{v}=\left[v_{1} \cdots v_{T N}\right]$. This block is divided into 2 layers and each layer contains $K N$ where $K=T / 2$. The space-time encoder constructs the codeword matrix $\mathbf{U}$ by inserting phase shifts between the symbols in the same layers and between the two layers as follows:

$$
\mathbf{U}=\left[\begin{array}{lllllll}
\mathbf{u}_{11} & \mathbf{u}_{12} & \mathbf{u}_{13} & \mathbf{u}_{14} & \cdots & \mathbf{u}_{1(N-1)} & \mathbf{u}_{1 N} \\
\mathbf{u}_{21} & \mathbf{u}_{22} & \mathbf{u}_{23} & \mathbf{u}_{24} & \cdots & \mathbf{u}_{2(N-1)} & \mathbf{u}_{2 N}
\end{array}\right]
$$

In (4), the column vectors $\mathbf{u}_{1, m}, \mathbf{u}_{1, m+1}, \mathbf{u}_{2, m}$ and $\mathbf{u}_{2, m+1}$ are given by

$$
\begin{aligned}
& \mathbf{u}_{1, m}=\left[\begin{array}{ll}
\mathbf{x}_{1, m} & \mathbf{x}_{1, m+1}
\end{array}\right]\left[\begin{array}{l}
1 \\
e^{j \alpha}
\end{array}\right] \\
& \mathbf{u}_{1, m+1}=\left[\begin{array}{ll}
\mathbf{x}_{2, m} & \mathbf{x}_{2, m+1}
\end{array}\right]\left[\begin{array}{l}
1 \\
e^{j \alpha}
\end{array}\right] \mathrm{e}^{\mathrm{j} \beta} \\
& \mathbf{u}_{2, m}=\left[\begin{array}{ll}
\mathbf{x}_{2, m} & \mathbf{x}_{2, m+1}
\end{array}\right]\left[\begin{array}{l}
1 \\
-e^{j \alpha}
\end{array}\right] \mathrm{e}^{\mathrm{j} \beta} \\
& \mathbf{u}_{2, m+1}=\left[\begin{array}{ll}
\mathbf{x}_{1, m} & \mathbf{x}_{1, m+1}
\end{array}\right]\left[\begin{array}{l}
1 \\
-e^{j \alpha}
\end{array}\right]
\end{aligned}
$$

where the frames $\mathbf{x}_{1, m}, \mathbf{x}_{1, m+1}, \mathbf{x}_{2, m}$ and $\mathbf{x}_{2, m+1}$ are column vectors each has $K$ symbols from vector $\boldsymbol{v}$ and $m=1,3,5, \cdots, N-1$. 
To maximize coding gain with full diversity order we have to maximize the minimum Euclidean distance between any two codewords $\mathbf{U}$ and $\mathbf{U}^{\prime}$. This can be done as follows. The root of the determinant of matrix $\left(\mathbf{U}-\mathbf{U}^{\prime}\right)\left(\mathbf{U}-\mathbf{U}^{\prime}\right)^{H}$ is the product of 2 distances $d_{1}$ and $d_{2}$ and it is given by

$$
D=\left|\prod_{n=1}^{2} d_{i}\right|
$$

Note that, for the codeword pairs that differ at only one diagonal in the codeword matrices, the pairwise coding gain is $G_{c}=D / 2$. Then the two angles $\alpha$ and $\beta$ are chosen to maximize the minimum distance between any two codeword matrices which leads to maximize the coding gain $G_{c}$ with full diversity order.

$$
\{\alpha, \beta\}=\arg \max \min _{\left\{\mathbf{U}, \mathbf{U}^{\prime}\right\}} D
$$

After applying the $K$-point Inverse Discrete Fourier Transform (IDFT) to each frame, the resultant codeword matrix $\mathbf{S}$ can be written as

$$
\mathbf{S}=\left[\begin{array}{cc}
\mathbf{F}^{-1} & \mathbf{0} \\
\mathbf{0} & \mathbf{F}^{-1}
\end{array}\right] \mathbf{U}
$$

where $\mathbf{F}$ is the $K$-point DFT matrix and -1 denotes matrix inverse. As can be noted the codeword matrix $\mathbf{S}$ contains $N$ OFDM frames on each antenna branch. A cyclic prefix of length $(L-1)$ is added to each OFDM frame.

At the receiver, after removing the first $L-1$ samples for each OFDM frame that corresponds to the cyclic prefix, $K$-point FFT is applied to each OFDM frame. This results in received signal matrix as

$$
\mathbf{Y}=\left[\begin{array}{cc}
\mathbf{F} & \mathbf{0} \\
\mathbf{0} & \mathbf{F}
\end{array}\right] \overline{\mathbf{H}}\left[\begin{array}{cc}
\mathbf{F}^{-1} & \mathbf{0} \\
\mathbf{0} & \mathbf{F}^{-1}
\end{array}\right] \mathbf{U}+\mathbf{V}
$$

with

$$
\overline{\mathbf{H}}=\left[\begin{array}{ll}
\overline{\mathbf{H}}_{11} & \overline{\mathbf{H}}_{12} \\
\overline{\mathbf{H}}_{21} & \overline{\mathbf{H}}_{22}
\end{array}\right]
$$

and $\overline{\mathbf{H}}_{j i}$ is $K \times K$ circulant matrix given as

$$
\overline{\mathbf{H}}_{j i}=\left[\begin{array}{cccccccc}
h_{j i}(0) & 0 & \cdots & 0 & & h_{j i}(L-1) & \cdots & h_{j i}(1) \\
h_{j i}(1) & h_{j i}(0) & 0 & \cdots & 0 & h_{j i}(L-1) & \cdots & h_{j i}(2) \\
\ddots & & & \ddots & & & & \\
& & & & & & & \\
0 & & \cdots & & 0 & h_{j i}(L-1) & \cdots & h_{j i}(0)
\end{array}\right]
$$

The received matrix $\mathbf{Y}$ can be written as

$$
\mathbf{Y}=\left[\begin{array}{ll}
\mathbf{F} \overline{\mathbf{H}}_{11} \mathbf{F}^{-1} & \mathbf{F} \overline{\mathbf{H}}_{12} \mathbf{F}^{-1} \\
\mathbf{F} \overline{\mathbf{H}}_{21} \mathbf{F}^{-1} & \mathbf{F} \overline{\mathbf{H}}_{22} \mathbf{F}^{-1}
\end{array}\right] \mathbf{U}+\mathbf{V}=\left[\begin{array}{cc}
\tilde{\mathbf{H}}_{11} & \tilde{\mathbf{H}}_{12} \\
\tilde{\mathbf{H}}_{21} & \tilde{\mathbf{H}}_{22}
\end{array}\right] \mathbf{U}+\mathbf{V}(15)
$$

where $\mathbf{V} \in C^{2 K \times N}$ is the additive complex Gaussian noise matrix with i.i.d entries, i.e., $v_{m, t} \sim C N\left(0, N_{0}\right)$ and $\tilde{\mathbf{H}}_{j i}$ is a diagonal matrix whose diagonal elements are the DFT coefficients of the vector

$\mathbf{h}_{j i}=\left[\begin{array}{llll}h_{j i}(0) & h_{j i}(1) & \cdots & h_{j i}(K-1)\end{array}\right]^{T}$. The above received matrix are sent to the optimum detector where the maximum likelihood decision rule is applied.

\section{Simulation Results}

In this section, we provide simulation results to compare the proposed scheme with the conventional schemes done by Hassibi [9] and Ma [10] in terms of performance. The channel model described in Section 2 with three equal-gain resolvable paths $(L=3)$ was used. Two transmit and two receive antennas were assumed. In all the schemes, two layers are employed with BPSK modulation and each OFDM frame has 32 symbols. For BPSK modulation, the optimized angles $\alpha$ and $\beta$ that maximizes the coding gain with full diversity were found to be $\alpha=\beta=\pi / 2$. It was mentioned before that Ma's scheme has full diversity and not maximized coding gain whereas Hassibi's scheme has not full diversity nor maximized coding gains. The decoding complexity of all schemes are comparable.

Figure 2 shows the Bit Error Rate (BER) as a function of Signal-to-Noise Ratio (SNR). It is seen that, at BER = $4 \times 10^{-5}$, a performance gain of approximately $5.7 \mathrm{~dB}$ is achieved for the proposed scheme over Hassibi's scheme and $3.9 \mathrm{~dB}$ over Ma's scheme. This result can be explained because Ma's scheme maximizes the diversity gain and preserves channel capacity but does not necessarily guarantee high coding gain which affects the performance of the system as discussed before. Although Hassibi scheme preserves channel capacity, it does not guarantee good performance since the diversity and cod-

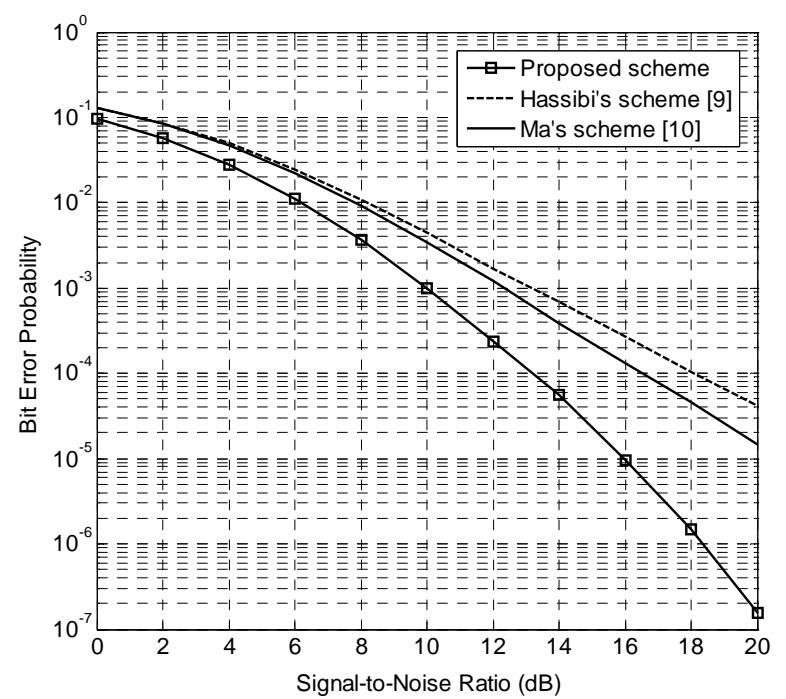

Figure 2. Bit error performance of Hassibi's scheme, Ma's scheme and the proposed scheme. 
ing gains were not explicitly optimized. As mentioned before, our proposed scheme preserves the channel capacity and maximizes both the diversity and coding gains.

\section{Conclusion}

In this paper, we have introduced a new OFDM spacetime coding scheme for quasi-static time dispersive MIMO channels. Due to the insertion of optimized phase shifts between the symbols in the same layers and between the two layers, the diversity and coding gains are maximized without loss of the capacity. This leads to improvement in the performance of the proposed scheme compared to the other conventional schemes. Simulation results have demonstrated to show the validity and effectiveness of the proposed scheme compared to others.

\section{REFERENCES}

[1] I. E. Telatar, "Capacity of Multi-Antenna Gaussian Channels," European Transaction on Telecommunications, Vol. 10, No. 6, 1999, pp. 585-595. doi:10.1002/ett.4460100604

[2] G. J. Foshini and M. J. Gans, "On the Limits of Wireless Communications in a Fading Environment When Using Multiple Antennas," Wireless Personal Communications, Vol. 6, No. 3, 1998, pp. 311-335. doi:10.1023/A:1008889222784

[3] V. Tarokh, N. Seshadri and R. Calderbank, "Space-Time Codes for High Data Rate Wireless Communication: Performance Criterion and Code Construction," IEEE Transaction on Information Theory, Vol. 44, No. 2, 1998, pp. 744-765. doi:10.1109/18.661517

[4] D. Agrawal, V. Tarokh, A. Naguib and N. Sheshadri, "Space-Time Coded OFDM for High Data-Rate Wireless Communication over Wideband Channels," IEEE Vehicu- lar Technology Conference, Vol. 3, Ottawa, 18-21 May 1998, pp. 2232-2236.

[5] S. M. Alamouti, "A Simple Transmit Diversity Technique for Wireless Communications," IEEE Journal on Selected Areas in Communications, Vol. 16, No. 8, 1998, pp. 14511458. doi:10.1109/49.730453

[6] V. Tarokh, H. Jafarkhani and A. R. Calderbank, "SpaceTime Block Codes from Orthogonal Designs," IEEE Transactions on Information Theory, Vol. 45, No. 5, 1999, pp. 1456-1467. doi:10.1109/18.771146

[7] S. Mudulodu and A. Paulraj, "A Transmit Diversity Scheme for Frequency-Selective Fading Channels," IEEE Global Telecommunications Conference, Vol. 2, San Francisco, 27 November-1 December 2000, pp. 1089-1093.

[8] S. Alkhawaldeh and Y. R. Shayan, "An Extended SpaceTime Transmit Diversity Scheme for Frequency Selective Fading Channels," IEEE CCECE Conference, Montreal, 12-15 May 2003, pp. 1663-1666.

[9] B. Hassibi and B. M. Hochwald, "High-Rate Codes that Are Linear in Space and Time," IEEE Transactions on Information Theory, Vol. 48, No. 7, 2002, pp. 1804-1824. doi:10.1109/TIT.2002.1013127

[10] X. Ma and G. B. Giannakis, "Full-Diversity Full-Rate Complex-Field Space-Time Coding," IEEE Transactions on Signal Processing, Vol. 51, No. 11, 2003, pp. $2917-$ 2930.

[11] R. W. Heath Jr. and A. J. Paulraj, "Linear Dispersion Codes for MIMO Systems Based on Frame Theory," IEEE Transactions on Signal Processing, Vol. 50, No. 10, 2002, pp. 2429-2441. doi:10.1109/TSP.2002.803325

[12] M. O. Damen, N. C. Beaulieu and J.-C. Belfiore, "A Number Theory Based Dual Transmit Antennas Space-Time Code," IEEE GLOBECOM Conference, San Antonio, 2529 November 2001, pp. 485-489.

[13] M. O. Damen, A. Tewfik and J.-C. Belfiore, "A Construction of a Space-Time Code Based on Number Theory," IEEE Transactions on Information Theory, Vol. 48, No. 3, 2002, pp. 753-760. doi:10.1109/18.986032 